\title{
Aprovechamiento integral del camarón de cultivo de la Bahía de Jiquilisco, departamento de Usulután, para su desarrollo industrial bajo normas de calidad e inocuidad
}

\author{
Ana Elizabeth Perlera de Escalante ${ }^{1}$ - Melba Jeannette Pacheco de Jordán ${ }^{2}$ - \\ Vilma Ruth Calderón de Zacatares ${ }^{3}$
}

Recepción: 29/04/017

Aceptación: 13/07/2017

\section{Resumen}

El objetivo de la investigación fue evaluar la producción y la caracterización fisicoquímica y microbiológica de tres tipos de harinas desarrolladas a partir de subproductos de camarón (Litopeneaus vannamei), originario de la Bahía de Jiquilisco; también se desarrollaron productos alimenticios elaborados con estas harinas. Además, se realizaron análisis microbiológicos de músculo abdominal crudo, exoesqueleto y hepatopáncreas, así como de las harinas formuladas. Se desarrollaron tres harinas a partir de cefalotórax y músculo abdominal, asimismo, siete productos alimenticios cuyo ingrediente principal son las harinas de músculo, cola y cabeza de camarón para evaluar la inocuidad de las harinas producidas, y tener parámetros para conocer la efectividad del uso del proceso de escaldado como reductor microbiano.

Palabras clave: Litopeneaus vannamei, productos alimenticios, inocuidad, proceso de escaldado.

\begin{abstract}
The objective of the investigation was to evaluate the production and the physical chemistry and microbiological characterization of three kinds of industrialized flour from sub-products of shrimp (Litopeneaus vannamei) from Jiquilisco Bay. Also, food products were developed made from these flours. Furthermore, microbiological analysis of natural abdominal muscle, exoskeleton and hepatopancreas as well as self-rising flour. There are three types of flour developed from the cephalothorax and abdominal muscle in the same way seven food products whose principal ingredients are the muscle flours, tail and shrimp head to evaluate the safety of the produced flour and have standards to know the efficiency of blanching process using as microbial reducer.
\end{abstract}

Key words: Litopeneaus vannamei, food products, safety, blanching process

1. Licenciada en Química y Farmacia con especialidad en Microbiología, Docente investigadora, Universidad Católica de El Salvador; email: ana.perlera@catolica.edu.sv

2. Técnica en Ingeniería Química, Docente investigadora, Universidad Católica de El Salvador; email: melba.pacheco@ catolica.edu.sv

3. Máster en Tecnología de alimentos, Investigadora, Parque Tecnológico en Agroindustria; email: vilma.calderon@mined.gob.sv 


\section{Introducción}

La camaronicultura actual de El Salvador es considerada de pequeña escala, según su perfil social, formas de trabajo y características técnicas de los sistemas de cultivo. Las cooperativas formadas por camaronicultores quedan en la categoría de Pequeñas y Medianas Empresas (PyME), específicamente en la Acuicultura de Micro y Pequeña Empresa (AMyPE), la cual carece en su mayor parte de tecnificación, sin conocimientos de cómo generar valor agregado, y sin gestión empresarial (Odonne y Beltrán, 2013).

En El Salvador, la producción de camarón es incipiente. De acuerdo a datos del Centro de Desarrollo de la Pesca y la Acuicultura del Ministerio de Agricultura y Ganadería de El Salvador (CENDEPESCA) ${ }^{4}$, en el 2010 se registraron veinticinco granjas camaroneras, todas ubicadas en la Bahía de Jiquilisco, departamento de Usulután, que es un sitio RAMSAR $^{5}$. De estas granjas, doce cultivan camarón de agua salada (Peneaus vannamei) y el resto camarón de agua dulce (Macrobrachium rosembergii).

La producción nacional estimada es de 3.3 millones de libras anuales, mientras que la producción pesquera es 907,200lb anuales (CENDEPESCA, 2015). En cuanto a la venta del producto, este es comprado por los intermediarios en borda, generalmente entero, fresco y sin tratamiento de desinfección previa, sin procesamiento mínimo o valor agregado. El manejo post cosecha, particularmente la cadena de frío, no es estandarizada, por lo que puede generar problemas de inocuidad, relacionadas al medio de comercialización como la sanitización previa a venta. Es usual que los intermediarios utilicen cajas isotérmicas con hielo en bloque para transportar y conservar el camarón hasta su sitio de venta (Odonne y Beltrán, 2013).

Los tamaños de camarones producidos en cultivo oscilan entre los 8 a $16 \mathrm{~g}$. El tamaño que los consumidores finales prefieren son de 8 a $10 \mathrm{~g}$, que por libra tienen de 41/50 camarones; y el tamaño de 11 a $12 \mathrm{~g}$, que por libra tiene de 36/40 camarones (calibre).

Los subproductos generados por la industria camaronera pueden dividirse en sólidos y líquidos. En cuanto a los sólidos encontramos: cefalotórax, cutícula o caparazón, vísceras y fragmentos de carne que no han sido removidos en la operación de pelado. Alrededor de un $40 \%$ de la utilización del camarón son desperdicios de cola, cabeza y caparazón, los cuales no tienen ningún uso posterior, produciendo contaminación ambiental, tanto de los desechos sólidos como de los líquidos (Calcedo, 2015).

4. Las autoras también se referirán a esta institución mediante sus siglas.

5. Se le denomina sitio RAMSAR a todos aquellos humedales de los cuales se hace uso racional y se les conserva mediante acciones locales y nacionales, gracias a la cooperación internacional, para una contribución al logro de un desarrollo sostenible en todo el mundo. 
Dentro de este estudio se desarrollaron procesos que ayudan a proporcionar metodologías y técnicas de procesamiento para el aprovechamiento integral de todas las partes del camarón y generación de valor agregado, poniendo a disposición de las cooperativas de camaroneros las tecnologías creadas, para que ellos puedan realizar un aprovechamiento integral del cultivo, y así competir en el mercado nacional e internacional. Además, se generó un precedente por las diferentes variables que pueden afectar el crecimiento microbiológico de los patógenos en estudio, y se verificó la inocuidad de los subproductos obtenidos del proceso de escaldado.

\section{Materiales y Métodos}

La investigación se realizó en las instalaciones del Parque Tecnológico Centroamericano PTA en el Laboratorio de Desarrollo de Prototipos Agroindustriales, en coordinación con la Universidad Católica de El Salvador (UNICAES), a través del personal del Laboratorio de Microbiología, en conjunto con los docentes de la escuela de Alimentos en la Facultad Multidisciplinaria de Ilobasco de la Universidad, con sede en el departamento de Cabañas.

La materia prima, en este caso el camarón de cultivo Litopeneaus vannamei, fue proporcionada por técnicos de Centro de Desarrollo de la Pesca y la Acuicultura (CENDEPESCA) través de la Unidad de Investigación acuícola que atiende las asociaciones de la Bahía de Jiquilisco. Se transportó, aún en estado fresco, en cajas isotérmicas; y luego fue mantenido en refrigeración a $0^{\circ} \mathrm{C}$ en el Laboratorio de Prototipos Agroindustriales del Parque Tecnológico en Agroindustria (PTA).

\section{Análisis microbiológicos en camarón fres- co y/o procesado}

Los análisis microbiológicos que se realizaron en muestras de camarón fresco fueron para la detección de Sthapylococcus aureus, Salmonella spp y Vibrio Parahaemolyticus. El método de cuantificación y/o determinación de presencia/ausencia que se utilizó fue el método de esparcido en placa para detección de Sthapylococcus aureus ${ }^{6}$, utilizando como medio selectivo Salt Egg Yolk Base y como medio confirmativo suero de conejo.

Para la determinación de Vibrio parahaemolyticus se utilizó la técnica de estriado en placa, tomando como diluyente agua peptonada alcalina (3\% de $\mathrm{NaCl})$ y la siembra en Agar TCBS ${ }^{7}$. Para la determinación de Salmonella $s p p^{8}$ se acondicionó la muestra en Caldo EEM como medio diluyente, utilizando como medio selectivo Selenito Cistina y Caldo Tetrationato; y como medio confirmativo Agar DHL, Agar MLCB. 
En lo que corresponde a los análisis de las harinas de cabeza y cola, y harina de músculo se utilizó el método en placa deshidratada marca 3M para determinación de Aerobios mesófilos, Hongos y levaduras, Salmonella spp y Escherichia coli ${ }^{9}$. Se realizó un muestreo en las Cooperativas Los Cálix y La Canoa del municipio de Jiquilisco, recolectando veintiún muestras al azar de un tanque; y el procedimiento de obtención de la muestra fue el siguiente: Se recolectó el camarón fresco en tiempo de cosecha; se trasladó en bolsa plástica, manteniendo la cadena de frío por medio de cajas isotérmicas previamente enfriadas con suficiente recurso. Al llegar al laboratorio se hizo un solo pull; posteriormente, se separaron las muestras sólo de músculo abdominal crudo (sin estómago y hepatopáncreas), hepatopáncreas y exoesqueleto. Por cada muestreo se clasificó en dos grupos para realizar los análisis correspondientes.

\section{Desarrollo de harinas de caparazón, mús-} culo y cola: Subproductos desarrollados

El personal del Parque Tecnológico Agroindustrial realizó la investigación pertinente para desarrollar metodologías para la transformación de caparazón, cola, cabeza y músculo de camarón en harinas para consumo humano y animal. Para ello se utilizaron muestras de camarón con calibre de 41/50 (8 a 10g peso), proveniente de la Cooperati- va Los Calix de la Bahía de Jiquilisco (sitio RAMSAR), a través de los técnicos de CENDEPESCA, procedente de la Cooperativa Los Calix de la Bahía de Jiquilisco, departamento de Usulután.

La materia prima utilizada fue camarón del genero Litopeneaus Vannamei. Además, se desarrollaron diferentes metodologías de acuerdo a las condiciones fisiológicas y morfológicas de este espécimen para la producción de harinas. Los parámetros de calidad que se determinaron en cada una de las harinas desarrolladas fueron:

\section{Calidad fisicoquímica y microbiológica}

\begin{tabular}{|c|}
\hline Variables \\
\hline Rendimiento de harina \\
\hline Humedad \\
\hline Granulometría \\
\hline Calidad microbiológica \\
\hline
\end{tabular}

Figura 1. Variables tomadas en cuenta respecto a la calidad fisicoquímica y microbiológica.

El experimento se desarrolló durante los meses de abril a diciembre del año 2016. Durante este periodo se hicieron varios test preliminares para determinar la mejor metodología que debía ser utilizada para la producción de harinas y para la caracterización fisicoquímica y microbiológica.

9. Método Oficial AOAC 


\section{Metodología para la elaboración de harinas de caparazón, cabeza y cola}

Inicialmente, los subproductos (exoesqueleto, cabeza y cola) fueron sujetos a un lavado con agua potable, donde se removieron algunas impurezas. Luego fueron sanitizados con agua clorinada al (0.5\%), sumergidos en hielo por veinte minutos.

Luego de este procedimiento las muestras, cada una por separado, fueron sometidas a procesos de escaldados por periodos de cinco, diez, quince y veinte minutos, consecutivamente, a $95^{\circ} \mathrm{C}$ para evaluar el mejor tiempo de pre cocción; y así evitar la desnaturalización de proteínas. El escaldado sirve para pre cocer el producto con la finalidad de remover el número de microorganismos patógenos o no, presentes en las superficies. Se tomaron muestras del agua de cocción; y la que presentó menor concentración de sólidos solubles totales en caldo fue la que se seleccionó.

Se realizaron pruebas de tiempos de secado en un deshidratador marca Nesco, por periodos de seis, ocho, diez y doce horas a $135^{\circ} \mathrm{F}$, para determinar el tiempo óptimo de secado, que redujera la humedad lo suficiente para inhibir el crecimiento de patógenos y aumentar la vida de anaquel del producto. Luego de someterse al secado, se midió la humedad en balanza para humedad modelo Ohaus para cada una de las materas primas en estudio. Tras el secado, las muestras se molieron en un molino de discos tipo Omega VI, elaborado por Tecnología Compatible Internacional (CTI). Las muestras fueron molidas a cuatro pasos, apretando discos en cada uno de ellos. Se realizaron análisis de granulometría para verificar el tamaño de partícula de las harinas y, nuevamente, análisis de humedad de cada uno de los subproductos.

Posteriormente, las muestras fueron empacadas en bolsas aluminizadas y resguardadas hasta su uso en la elaboración de productos alimenticios, y observar la vida de anaquel.

\section{Vida de anaquel}

Después de empacadas fueron colocadas en anaquel bajo condiciones promedio de temperatura de 33 a $35^{\circ} \mathrm{C}$ y humedad relativa de 60-65\%. Luego fueron sometidas a observación visual y destrucción mensual de muestras elaboradas para realizar análisis fisicoquímico y microbiológico. Las harinas fueron observadas por un periodo de seis meses.

\section{Metodología para la elaboración de harina de músculo de camarón}

Para la elaboración de harina de músculo abdominal, se siguieron los mismos pasos que para la elaboración de harinas de cabeza, cola y caparazón (exoesqueleto); a excepción de la desinfección con cloro. 


\section{Resultados y Discusión}

\section{a. Harinas de camarón y subproductos}

\section{Desarrollo de harina de cabezas}

Después de realizar las pruebas preliminares se determinó que la metodología para el desarrollo de esta harina comprende los siguientes pasos:

- Lavado y desinfección con agua potable y solución clorada al (0.5\%)

- Escaldado por ocho minutos en agua en ebullición a $95^{\circ} \mathrm{C}^{10 * *}$

- Escurrido en colador por diez minutos

- Pesado de muestra

- Deshidratado por ocho horas en deshidratador eléctrico marca Nesco Food Dehydrator a $135^{\circ} \mathrm{F}$
- Molienda a cuatro pasos en molino de discos

- Empacado en bolsa aluminizada y almacenado a temperatura ambiente

Los sólidos solubles fueron de un $12.7 \%$ y la pigmentación del agua presentó coloración en valores RGB de 255, 169 y 113 . La vida de anaquel estimada de producto fue de cuatro meses, bajo condiciones normales de humedad y temperatura.

\section{Caracterización y rendimiento promedio} de harina de cabezas de camarón

Los resultados obtenidos en cuanto a la caracterización de la harina de cabezas se resumen en la tabla siguiente:

Tabla 1. Caracterización de la harina de cabezas de camarón Litopeneaus vannamei

\begin{tabular}{|c|c|c|c|c|}
\hline Humedad & Granulometría & Colorimetría & Rendimiento & $\begin{array}{c}\text { Aplicaciones en agro- } \\
\text { industria }\end{array}$ \\
\hline $3.94 \%$ & $\begin{array}{c}0.25 \text { a } 0.60 \\
(\text { mesh } 60)\end{array}$ & $22.5 \%$ & $\begin{array}{c}\text { Elaboración de conso- } \\
\text { més, condimentos, sopas, } \\
\text { cremas y alimentos con- } \\
\text { centrados para animales }\end{array}$ \\
\hline
\end{tabular}

10. Para un tiempo de escaldado de ocho minutos. 
La harina de cabezas está formada por parte de exoesqueleto, compuesto de Quitina y Quitosano, principalmente, y por partes orgánicas; esto hace mucho más difícil el tiempo de secado para poder reducir la humedad a niveles aceptables. Según Ramos et al, (2008), las harinas de cabeza que se usan para balancear concentrado para animales, luego de un secado por diez horas, alcanzan una humedad del 3.5\%; lo cual ayuda a alargar la vida de anaquel del producto. La concentración de sólidos solubles totales en aguas de escaldado indica el grado de pigmentos astaxantinas y otras sustancias que se desnaturalizan durante la cocción.

Calcedo M. (2005), afirma que las aguas de cocción de camarones o aguas de blanqueo alcanzan concentraciones de sólidos solubles de hasta $15 \%$ cuando los tiempos no son los adecuados. Esta característica se debe aprovechar para el desarrollo y formulación de alimentos como sopas, caldos, consomés y sazonadores.

Los rendimientos de harina son menores, debido a que las concentraciones de agua en tejidos orgánicos son mucho mayores; de ahí se deduce la pérdida de peso, datos que coinciden con los reportados por Fernández et al. (2013).

\section{Desarrollo de harina de caparazón (exoes- queleto) y cola de camarón}

Posterior a realizar las pruebas preliminares, se determinó que la metodología para el desarrollo de esta harina comprende los siguientes pasos:

- Lavado y desinfección con agua potable y solución clorada al (0.5\%)

- Escaldado por cinco minutos en agua en ebullición a $95^{\circ} \mathrm{C}^{11}$

- Escurrido en colador por diez minutos

- Pesado de muestra

- Deshidratado por cuatro horas en deshidratador eléctrico marca Nesco Food Dehydrator a $135^{\circ} \mathrm{F}$

- Molienda a cuatro pasos en molino de discos

- Empacado en bolsa aluminizado y almacenado a temperatura ambiente

Los sólidos solubles fueron de un $11.3 \%$ y la pigmentación del agua presentó coloración en valores RGB de 243, 161 y 60.

El estimado de vida en anaquel fue de seis meses, bajo condiciones normales de empaque, humedad y temperatura.

11. Para un tiempo de escaldado de cinco minutos. 
Caracterización y rendimiento promedio de harina de exoesqueleto y cola

Los resultados de caracterización para la harina de exoesqueleto y cola de camarón se resumen en la tabla 2.

Para este tipo de harina, la concentración de sólidos solubles en caldo fue menor, ya que el exoesqueleto está formado por compuestos de Quitosano y Quitina, los cuales son insolubles en agua y pigmentos astaxantinicos solubles en agua; por lo que se deduce que la concentración de pigmentos es la que concentra el agua de escaldado. Asimismo, la humedad fue menor, ya que no existe suficiente agua libre que pueda liberarse. Ramos et. al, (2008), reporta que el exoesqueleto de camarón, contiene $15 \%$ de agua libre, lo cual coincide con lo manifestado. Debido a ello también puede modificarse los rendimientos de la harina, los cuales aumentan debido a la pérdida de peso en agua y es del 26\%. El tamaño de partícula o datos granulométricos se mantiene.
La estimación de la vida útil del producto se puede atribuir al bajo contenido de humedad luego del deshidratado; además, al bajo contenido de grasas u otros componentes.

\section{Desarrollo y caracterización de harina de músculo abdominal}

Para la obtención de harina de músculo abdominal se determinaron los siguientes pasos:

- Lavado

- Escaldado por doce minutos en agua en ebullición a $95^{\circ} \mathrm{C}^{12}$

- Escurrido en colador por diez minutos

- Pesado de muestra

- Deshidratado por doce horas en deshidratador eléctrico marca Nesco Food Dehydrator a $135^{\circ} \mathrm{F}$

- Molienda a tres pasos en molino de discos

- Empacado en bolsa aluminizado y almacenado a temperatura ambiente

Tabla 2. Caracterización de harina de exoesqueleto de camarón Litopeneaus vannamei

\begin{tabular}{|c|c|c|c|c|}
\hline Humedad & Granulometría & Colorimetría & Rendimiento & $\begin{array}{c}\text { Aplicaciones en agro- } \\
\text { industria }\end{array}$ \\
\hline $2.3 \%$ & $\begin{array}{l}0.50 \text { a } 0.70 \\
(\text { mesh } 60)\end{array}$ & & $73.9 \%$ & $\begin{array}{l}\text { Alimentación animal. } \\
\text { Elaboración de concentra- } \\
\text { dos para tilapia, tortugas y } \\
\text { camarones. Alimentación } \\
\text { de bovinos y porcinos en } \\
\text { formulas balanceadas. }\end{array}$ \\
\hline
\end{tabular}

12. Para un tiempo de escaldado de cinco minutos 
Los sólidos solubles fueron de un 7.3\% y la pigmentación del agua presentó coloración en valores RGB de 255, 116 y 48. La vida de anaquel estimada de la harina de músculo abdominal es de seis meses, bajo las condiciones normales de humedad, temperatura y empaque, (ver tabla 3).

La harina de músculo de camarón se tipifica por contener menor cantidad de pigmentos, además de una humedad mayor a las demás harinas, ya que está constituida por tejido muscular que contiene una mayor cantidad de agua libre y agua ligada (Aw). Los resultados que coinciden con Calcedo M. (2005), quien en su estudio sobre aprovechamiento de los desechos del camarón en la elaboración de concentrados proteicos y derivados quitinosos, manifiesta haber obtenido parámetros de humedad y granulometría similares, debido a las condiciones anteriormente mencionadas, de humedad y concentración de pigmentos, la concentración de sólidos solubles en caldo de escaldado fue de 7.3\%. La reducción de la humedad de la harina y por ende de la actividad del agua (Aw) es crítico para aumentar la vida de anaquel.

La granulometría o tamaño de partícula fue menor, o sea de tamaño más pequeño que las demás harinas, facilitando la molienda, haciéndola únicamente de tres pasos en molino de discos.

\section{Resultados de análisis microbiológicos de camarón fresco}

Se analizaron un total de veintiún muestras tomadas al azar de las cuales solo una presentó crecimiento de Escherichia coli de 126 $\mathrm{UFC/g}$ de una muestra de exoesqueleto, las demás muestras presentaron crecimiento menor de $60 \mathrm{UFC/g}$. Se encontró Vibrio parahaemolitycus en cuatro muestras; sin embargo, seis muestras presentaron crecimiento característicos por morfología, típicos del Vibrio algenoliticus, con colonias amarillas aplanadas sin mostrarse correspondientes

Tabla 3. Caracterización y rendimiento promedio de harina de músculo abdominal

\begin{tabular}{|c|c|c|c|}
\hline Humedad & Granulometría & Rendimiento & $\begin{array}{c}\text { Aplicaciones en agro- } \\
\text { industria }\end{array}$ \\
\hline $5.3 \%$ & $\begin{array}{c}0.30 \text { a } 0.50 \\
(\text { mesh } 80)\end{array}$ & $69 \%$ & $\begin{array}{c}\text { Alimentación humana. } \\
\text { Elaboración de snack, ga- } \\
\text { lletas. Condimentos, sazo- } \\
\text { nadores, cremas y sopas. }\end{array}$ \\
\hline
\end{tabular}


a la tinción gram, tampoco a las pruebas de Oxidasa y Catalasa, por lo que no se tomaron como significativas. Solamente una de las muestras tenía presencia de Staphylococcus aureus y para Aerobios mesófilos se encontraron diecisiete muestras con presencia, pero de estas ocho estaban arriba de 4,000 UFC/g, (ver figura 2).

\section{Resultados microbiológico en harinas}

De las tres muestras analizadas no hubo crecimiento de Salmonella spp, Staphylococcus aureus, Escherichia coli y el de hongos y levaduras. En el caso de Aerobios mesófilos hubo crecimiento en todas las muestras, pero ninguna excedió las 10UFC/g que establece el RTCA 67.04.50:08 sobre Alimentos, Cri- terios Microbiológicos para la Inocuidad de Alimentos, Clasificación 9.2 Subgrupo del alimento: Pescado y crustáceos, pre-cocidos, cocidos, salados y ahumados. A las dos semanas de realizados los primeros análisis se volvieron a hacer con las muestras en retención obteniendo los mismos resultados en Salmonella spp, Staphylococcus aureus, Escherichia coli y el de hongos y levaduras, pero en Aerobios mesófilos hubo un crecimiento del 10\% mayor que los resultados anteriores, pero siempre dentro de los parámetros establecidos por norma.

\section{Valor agregado en productos de camarón}

A medida que la producción de camarón de cultivo crezca, día a día va a tener mayor re-

Resutados de Análisis de Aerobios mesófilos

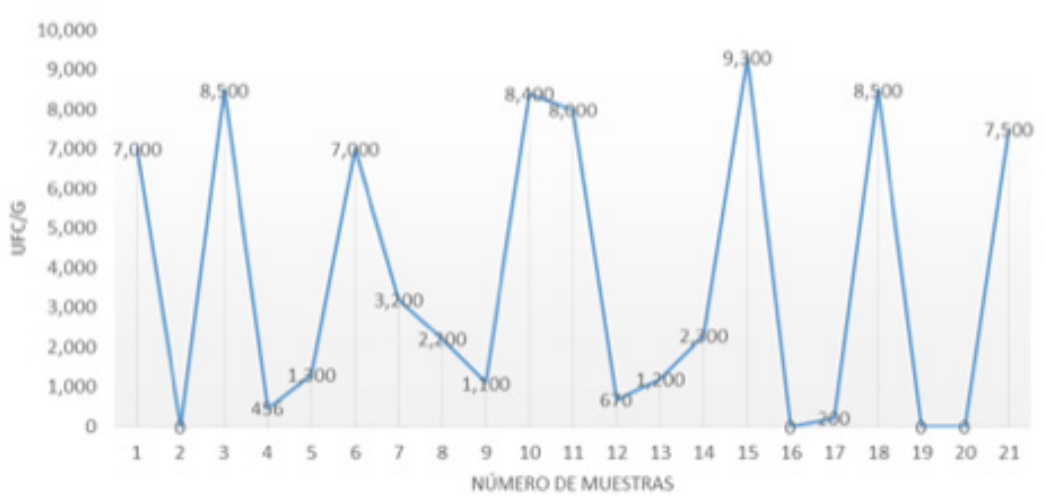

Figura 2. Resultados de Análisis de Aerobios mesófilos en camarón en fresco. 
levancia el concepto de valor agregado. Los grandes exportadores de camarones en la región como Ecuador y Tailandia han encontrado desde los años 90 una alternativa para mover su producción con mayor rentabilidad. No es lo mismo ofrecer camarón fresco con cáscara; que realizar algunas formas de procesamiento mínimo como pelado y desvenado, corte mariposa y empacados al vacío etc.; o también desarrollar nuevos productos cuyo ingrediente principal o secundario sean las harinas elaboradas con subproductos del camarón. En general, el objetivo del valor agregado es aumentar la competitividad y rentabilidad.

Con las harinas formuladas se desarrollaron diferentes tipos de productos entre los que figuran snack, galletas, sopas, cremas y productos cárnicos. Se utilizaron en sus formulaciones diferentes porcentajes de harinas tanto de cabeza como de músculo abdominal. En la siguiente tabla se muestran los diferentes productos formulados con las diferentes harinas y algunas de sus características:

Tabla 4. Productos alimenticios formulados y porcentajes de harina utilizados

\begin{tabular}{|c|c|c|}
\hline Producto Formulado & Tipo(s) de harina utilizada & $\begin{array}{c}\text { Porcentaje de harina usada } \\
\text { en producto }\end{array}$ \\
\hline Consomé & Harina de cabeza & $15 \%$ \\
\hline Crema de camarones & Harina de músculo & $20 \%$ \\
\hline Sopa Tipo Ramen & Harina de cabeza & $15 \%$ \\
\hline Sazonador & Harina de músculo abdominal & $15 \%$ \\
\hline Tortilla chips & Harina de músculo abdomi- & $10 \%$ harina de cabeza \\
\hline Galletas tipo Ritz & nal y harina de cabezas & $10 \%$ harina de músculo \\
\hline Nuggets con coco & Harina de músculo & $10 \%$ harina de cabeza \\
\hline Palitos verdes con camarón & Harina de músculo y harina & $10 \%$ harina de músculo \\
\hline Chorizos de camarón & de cabeza & $25 \%$ \\
\hline & Harina de músculo & $5 \%$ harina de músculo \\
\hline & Harina de cabezas \\
\hline
\end{tabular}




\section{Conclusiones}

Es factible técnicamente usar subproductos de camarón (Litopeneaus vannamei) como cabezas, exoesqueleto, (caparazón y cola); además de músculo abdominal para la elaboración de harinas de consumo humano y animal.

Las harinas de subproductos presentan buenas características fisicoquímicas para la elaboración de diferentes tipos de alimentos. El proceso de escaldado al que fueron sometidos los camarones para la obtención de harinas baja los niveles de carga microbiológica significativamente, y debido a ello los resultados microbiológicos realizados a las harinas dieron como resultados muestras inocuas.
El 81\% de las muestras de camarón fresco dieron como resultado la presencia de $A e$ robios mesófilos, por lo que es necesario garantizar la cadena de frío en el transporte y almacenamiento de los camarones. En cuanto al resto de pruebas microbiológicas, el 10\% de las muestras tenían presencia de Escherichia coli y Sthapylococcus aureus y 19\% tenían presencia de Vibrio parahaemolyticus. Parte fundamental para esta investigación fue el apoyo y la información proporcionada por el Centro de Desarrollo de la Pesca y la Acuicultura del Ministerio de Agricultura y Ganadería en cuanto a información de las cooperativas de la Bahía de Jiquilisco.

\section{Referencias}

Caicedo, M. (2005). Aprovechamiento de los desechos del camarón en la elaboración de concentrados proteicos y derivados quitinosos. [Tesis doctoral]: Universidad del Magdalena: Universidad del Magdalena.

Camacho, R.; Méndez, E. y otros. (2014). Evaluación de la presencia de Vibrio parahaemolyticus en camarón blanco (Litopenaeus vannamei) silvestre estuarino en el sur de Sinaloa y norte de Nayarit, mediante análisis microbiológico y PCR. Recuperado de http://biociencias.uan.edu.mx/publicaciones/04-04/biociencias4-4-6.pdfç

Doyle, P. (2001). Microbiología de los Alimentos, bacterias patógenas. Editorial Acribia

El Salvador, Ministerio de Salud Pública y Asistencia Social (1997). Reglamento Técnico Centroamericano, RTCA 67.04.50:08. Alimentos, criterios microbiológicos para inocuidad de alimentos; 9.1 y 9.2, subgrupo: pescado y productos pesqueros frescos, congelados, moluscos, crustáceos y equinodermos empacados. Recuperado de https:// members.wto.org/crnattachments/2008/sps/CRI/08_1142_00_s.pdf 
El Salvador, Ministerio de Relaciones Exteriores, Banco de Desarrollo de América Latina (2013). Cooperación Regional en el Ámbito de la Integración Fronteriza San Salvador. Recuperado de http://www20.iadb.org/intal/catalogo/PE/2013/11724a05.pdf

Leiva, V.; Cisneros, E. y otros (1998). Aislamiento de Salmonellas atípicas en camarones congelados. Recuperado de http://bvs.sld.cu/revistas/ali/vol12_1_98/ali02198.htm

Leyton, Y. y Riquelme, C. (2008). Vibrio en los sistemas marinos costeros

Méndez, F. T.; da Silva, J.A.; Oliveira e C, J.M. y da Conceiao, M.L., (2013). Flour Production from shrimp by-products and sensory evaluation of flour-based products. Psq. Agropec. Bras. Brasilia vol. 48; pp. 962-967

Odonne, N. y Beltrán, S. (2013). Diagnóstico de la cadena del camarón de cultivo. XXIV Reunión de Directores de Cooperación Internacional de América Latina y el Caribe

Omoe, K; y otros (2003). Characterization of Novel Staphylococcal Enterotoxine-Like toxin type “P”. Infect Immune 711, pp. 60-88

Ramos, R.; Miranda, I. y Molina, C. (2008). Consumo y digestibilidad aparente de tres ingredientes marinos locales incorporados en dietas prácticas para el camarón blanco Litopeneaus vannamei, Estudios Oceanológicos, vol. 20; pp. 43-44 